\title{
Investigation of eigenvibrations of a loaded bar
}

\author{
Anton A. Samsonov ${ }^{1}$, and Sergey I. Solov'ev ${ }^{1, *}$ \\ ${ }^{1}$ Kazan Federal University, 18 Kremlevskaya Street, Kazan 420008, Russian Federation
}

\begin{abstract}
The differential eigenvalue problem describing eigenvibrations of a bar with fixed ends and attached load at an interior point is investigated. This problem has an increasing sequence of positive simple eigenvalues with limit point at infinity. To the sequence of eigenvalues, there corresponds a complete orthonormal system of eigenfunctions. We formulate limit differential eigenvalue problems and prove the convergence of the eigenvalues and eigenfunctions of the initial problem to the corresponding eigenvalues and eigenfunctions of the limit problems as load mass tending to infinity. The original differential eigenvalue problem is approximated by the finite difference method on a uniform grid. Error estimates for approximate eigenvalues and eigenfunctions are established. Theoretical results are illustrated by numerical experiments for a model problem. Investigations of this paper can be generalized for the cases of more complicated and important problems on eigenvibrations of beams, plates and shells with attached loads.
\end{abstract}

\section{Introduction}

Let us formulate the differential eigenvalue problem governing eigenvibrations of the barload system. Assume that the elastic bar axis occupies in the equilibrium position the segment $[0, l]$ on the $O x$ axis. Denote by $\rho(x)$ the volume mass density, $E(x)$ the elasticity Young modulus, $S(x)$ the square of transversal cut of the bar. Suppose that the ends $x=0$ and $x=l$ of the bar are rigidly fixed, at a point $x^{(1)} \in(0, l)$ of the bar a load of mass $M$ is rigidly joined. Then the longitudinal deflection $w(x, t)$ of the bar at a point $x$ at time $t$ satisfies the following equations

$$
\begin{gathered}
\frac{\partial}{\partial x}\left(p(x) \frac{\partial}{\partial x} w(x, t)\right)=r(x) \frac{\partial^{2}}{\partial t^{2}} w(x, t), x \in\left(0, x^{(1)}\right) \cup\left(x^{(1)}, l\right), t>0, \\
w(0, t)=0, w(l, t)=0, t>0, \\
{[w(x, t)]_{x=x^{(1)}}=0,\left[p(x) \frac{\partial}{\partial x} w(x, t)\right]_{x=x^{(1)}}=M \frac{\partial^{2}}{\partial t^{2}} w\left(x^{(1)}, t\right), t>0,}
\end{gathered}
$$

\footnotetext{
*Corresponding author: sergei.solovyev@kpfu.ru
} 
where $[w(x, t)]_{x=x^{(1)}}=w\left(x^{(1)}+0, t\right)-w\left(x^{(1)}-0, t\right), \quad p(x)=E(x) S(x), \quad r(x)=\rho(x) S(x)$, $x \in[0, l], t>0$.

The eigenvibrations of the bar-load mechanical system are characterized by the function $w(x, t)$ of the following form $w(x, t)=u(x) \sin (\omega t), \quad x \in[0, l], t>0$, where $\omega$ is a constant. The number $\omega$ determines the eigenvibration frequency of the bar-load system, the function $u(x), x \in[0, l]$, determines the eigenvibration amplitude at each point $x \in[0, l]$ of the bar, and the function $\sin (\omega t), t>0$, describes the motion in time of points of the bar-load mechanical system under the eigenvibration.

Equations (1)-(3) lead to the following eigenvalue problem: find numbers $\lambda=\lambda(M)$ and nonzero functions $u(x)=u^{M}(x), x \in[0, l]$, such that

$$
\begin{gathered}
-\left(p(x) u^{\prime}(x)\right)^{\prime}=\lambda r(x) u(x), x \in\left(0, x^{(1)}\right) \cup\left(x^{(1)}, l\right), \\
u(0)=0, u(l)=0,[u(x)]_{x=x^{(1)}}=0,\left[p(x) u^{\prime}(x)\right]_{x=x^{(1)}}=-\lambda M u\left(x^{(1)}\right) .
\end{gathered}
$$

Problem (4), (5) has an increasing sequence of positive simple eigenvalues with limit point at infinity. To the sequence of eigenvalues, there corresponds a complete orthonormal system of eigenfunctions. In the present paper, limit properties as $M \rightarrow \infty$ of eigenvalues and eigenfunctions of problem (4), (5) are studied. To illustrate theoretical results, the initial problem is approximated by the finite difference method on a uniform grid. The errors of approximate eigenvalues and eigenfunctions are studied.

Parameter dependent eigenvalue problems are applied for investigating and solving nonlinear eigenvalue problems. Nonlinear eigenvalue problems have numerous applications in science and engineering [1-5]. Computational methods for solving nonlinear matrix eigenvalue problems were studied in [6-14]. The error of the finite difference method for solving differential eigenvalue problems with nonlinear dependence on the spectral parameter was investigated in $[1,15]$. For nonlinear differential spectral problems, the finite element method was studied in [16-19] based on the use general results in the linear case [20-23]. Approximate methods for solving applied nonlinear boundary value problems and variational inequalities have been investigated in the papers [24-30].

\section{Limit properties of the bar-load eigenvalue problem}

Put $\Omega=(0, l)$ and $\bar{\Omega}=[0, l]$. Define sufficiently smooth functions $p(x), r(x), x \in \bar{\Omega}$, for which there exist positive constants $p_{i}, \quad r_{i}, \quad i=1,2$, such that $p_{1} \leq p(x) \leq p_{2}$, $r_{1} \leq r(x) \leq r_{2}, x \in \bar{\Omega}$. Introduce positive numbers $M, x^{(1)} \in \Omega$, and bilinear forms

$$
a(u, v)=\int_{0}^{l} p(x) u^{\prime}(x) v^{\prime}(x) \mathrm{d} x, b(u, v)=\int_{0}^{l} r(x) u(x) v(x) \mathrm{d} x, c(u, v)=u\left(x^{(1)}\right) v\left(x^{(1)}\right) .
$$

Problem (4), (5) has an increasing sequence of positive simple eigenvalues with limit point at infinity $\lambda_{k}=\lambda_{k}(M), k=1,2, \ldots$ These eigenvalues correspond to an orthonormal system of eigenfunctions $\quad u_{k}=u_{k}^{M}, \quad k=1,2, \ldots, \quad a\left(u_{i}, u_{j}\right)=\lambda_{i} \delta_{i j}$, $b\left(u_{i}, u_{j}\right)+M c\left(u_{i}, u_{j}\right)=\delta_{i j}, i, j=1,2, \ldots$ 
Introduce the following limit problem: find numbers $\mu$ and nonzero functions $u(x)$, $x \in[0, l]$, such that

$$
\begin{gathered}
-\left(p(x) u^{\prime}(x)\right)^{\prime}=\mu r(x) u(x), x \in\left(0, x^{(1)}\right) \cup\left(x^{(1)}, l\right), \\
u(0)=0, u\left(x^{(1)}\right)=0, u(l)=0 .
\end{gathered}
$$

Problem (7), (8) has an increasing sequence of positive simple eigenvalues with limit point at infinity $\mu_{i}, \quad i=2,3, \ldots$ These eigenvalues correspond to an orthonormal systems of eigenfunctions $v_{i}, i=2,3, \ldots$ Set $\mu_{1}=0$.

Introduce the following limit eigenvalue problem: find numbers $\eta$ and nonzero functions $v(x), x \in[0, l]$, such that

$$
\begin{gathered}
-\left(p(x) v^{\prime}(x)\right)^{\prime}=0, x \in\left(0, x^{(1)}\right) \cup\left(x^{(1)}, l\right), \\
v(0)=0, v(l)=0,[v(x)]_{x=x^{(1)}}=0,\left[p(x) v^{\prime}(x)\right]_{x=x^{(1)}}=-\eta v\left(x^{(1)}\right) .
\end{gathered}
$$

Problem (9), (10) has one eigenvalue $\eta$ which is positive and simple. There exists a unique normalized positive eigenfunction $v_{1}(x), x \in[0, l]$, corresponding to the eigenvalue $\eta$.

Theorem 1. The following limit properties hold: $\lambda_{k}(M) \rightarrow \mu_{k}$ as $M \rightarrow \infty$, $\lambda_{1}(M) M \rightarrow \eta$ as $M \rightarrow \infty,\left\|u_{k}^{M}-v_{k}\right\| \rightarrow 0$ as $M \rightarrow \infty$, where $\|v\|=\max _{x \in \bar{\Omega}}|v(x)|$.

The proof of this theorem generalizes results from [4].

\section{Numerical experiments}

Introduce the partition of the segment $[0, l]$ by equidistant points $x_{i}=i h, i=0,1, \ldots, N$, $h=l / N$. Assume that $x^{(1)}=x_{m}$ for some $m, 0<m<N$. Denote $\tilde{p}_{i}=p\left(x_{i}-h / 2\right)$, $r_{i}=r\left(x_{i}\right), i=0,1, \ldots, N, \quad \delta_{i i}=1, \quad \delta_{i j}=0, i \neq j, \quad y_{x, i}=\left(y_{i+1}-y_{i}\right) / h, \quad y_{\bar{x}, i}=\left(y_{i}-y_{i-1}\right) / h$, $i=1,2, \ldots, N-1$. Approximate problem (4), (5) by the following finite difference scheme

$$
\begin{gathered}
\left(\tilde{p} y_{\bar{x}}\right)_{x, i}=\lambda^{h}\left(r_{i}+\frac{\delta_{m i}}{h} M\right) y_{i}, i=1,2, \ldots, N-1, \\
y_{0}=0, y_{N}=0 .
\end{gathered}
$$

Problem (11), (12) has eigenvalues $\lambda_{1}^{h}<\lambda_{2}^{h}<\ldots<\lambda_{N-1}^{h}$ and eigenvectors $y=y^{(k)}$, $k=1,2, \ldots, N-1, \quad \sum_{i=1}^{N-1} r_{i} y_{i}^{2}+M y_{m}^{2}=1$.

Theorem 2. The following error estimates are valid: $\left|\lambda_{k}^{h}-\lambda_{k}\right| \leq c h^{2}$, $\left\|y^{(k)}-u_{k}\right\|_{h} \leq c h^{2}$, where $c$ is a positive constant independent of $h$, $\left\|y^{(k)}-u_{k}\right\|_{h}=\max _{i=1,2, \ldots, N-1}\left|y_{i}^{(k)}-u_{k}\left(x_{i}\right)\right|$.

The proof of this theorem generalizes results from [23]. 
To illustrate theoretical results of Theorem 1, we have solved the eigenvalue problem (4), (5) for $p(x)=1, \quad r(x)=1, x \in[0, l], \quad l=1, x^{(1)}=0.3, \quad M \in[0,2]$, applying the finite difference scheme (11), (12) for $N=100$. Fig. 1 shows the graphs of the functions $\lambda_{i}(M)$, $M \in[0,2], i=1,2, \ldots, 6$, the eigenvalues $\mu_{i}, i=2,3, \ldots, 6$, of the limit linear eigenvalue problem (7), (8). We see that the experimental results are consistent with the theoretical results of Theorem 1; namely, $\lambda_{k}(M) \rightarrow \mu_{k}$ as $M \rightarrow \infty$. Investigations of the present paper can be generalized for the problems on eigenvibrations of beams, plates and shells with attached loads.
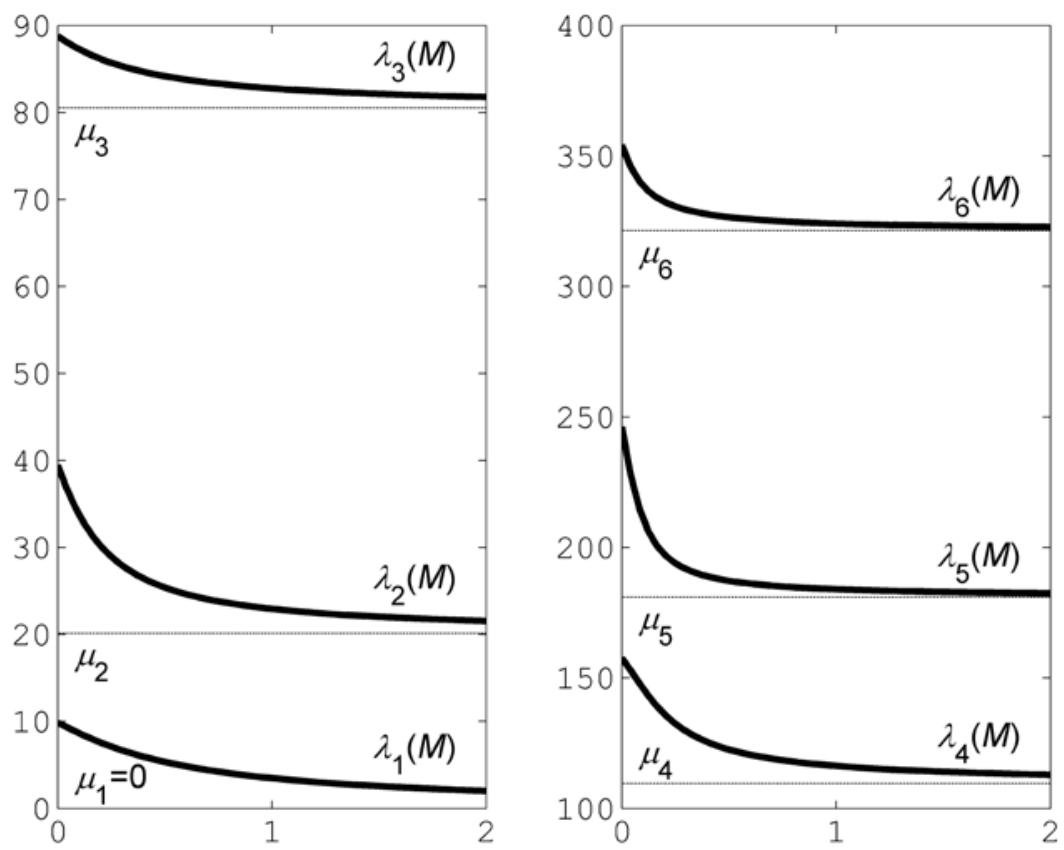

Fig. 1. Limit properties of the bar-load eigenvalue problem.

This work was supported by Russian Science Foundation, project no. 16-11-10299.

\section{References}

1. A.V. Goolin, S.V. Kartyshov, Surv. Math. Ind. 3, 29-48 (1993)

2. T. Betcke, N.J. Higham, V. Mehrmann, C. Schröder, F. Tisseur, ACM Trans. Math. Software 39, 7 (2013)

3. V.A. Kozlov, V.G. Maz'ya, J. Rossmann, Spectral Problems Associated with Corner Singularities of Solutions to Elliptic Equations (AMS, Providence, 2001)

4. S.I. Solov'ev, Lobachevskii J. Math. 37, 597-609 (2016)

5. S.I. Solov'ev, Izv. Vyssh. Uchebn. Zaved. Mat. 10, 71-74 (1985)

6. A. Ruhe, SIAM J. Numer. Anal. 10, 674-689 (1973)

7. V. Mehrmann, H. Voss, GAMM-Mit. 27, 1029-1051 (2004)

8. F. Tisseur, K. Meerbergen, SIAM Rev. 43, 235-286 (2001)

9. D. Kressner, Numer. Math. 114, 355-372 (2009) 
10. X. Huang, Z. Bai, Y. Su, J. Comput. Math. 28, 218-234 (2010)

11. H. Schwetlick, K. Schreiber, Linear Algebra Appl. 436, 3991-4016 (2012)

12. W.-J. Beyn, Linear Algebra Appl. 436, 3839-3863 (2012)

13. A. Leblanc, A. Lavie, Eng. Anal. Bound. Elem. 37, 162-166 (2013)

14. X. Qian, L. Wang, Y. Song, J. Comput. Appl. Math. 290, 268-277 (2015)

15. A.V. Kregzhde, Differ. Uravn. 17, 1280-1284 (1981)

16. S.I. Solov'ev, Comput. Math. Math. Phys. 32, 579-593 (1992)

17. R.Z. Dautov, A.D. Lyashko, S.I. Solov'ev, Differ. Equations 27, 799-806 (1991)

18. S.I. Solov'ev, Differ. Equations 50, 947-954 (2014)

19. S.I. Solov'ev, Differ. Equations 51, 934-947 (2015)

20. S.I. Solov'ev, Differ. Equations 30, 1138-1146 (1994)

21. S.I. Solov'ev, Differ. Equations 38, $752-753$ (2002)

22. S.I. Solov'ev, Differ. Equations 48, 1028-1041 (2012)

23. S.I. Solov'ev, Appl. Numer. Math. 93, 206-214 (2015)

24. I.B. Badriev, G.Z. Garipova, M.V. Makarov, V.N. Paymushin, Res. J. Appl. Sciences 10, 428-435 (2015)

25. I.B. Badriev, L.A. Nechaeva, PNRPU Mech. Bull. 3, 37-65 (2013)

26. I.B. Badriev, M.V. Makarov, V.N. Paimushin, Proc. Engin. 150, 1050-1055 (2016)

27. I.B. Badriev, G.Z. Garipova, M.V. Makarov, V.N. Paimushin, R. F. Khabibullin, Lobachevskii J. Math. 36, 474-481 (2015)

28. I.B. Badriev, M.V. Makarov, V.N. Paimushin, Russ. Math. 59, 57-60 (2015)

29. I.B. Badriev, M.V. Makarov, V.N. Paimushin, Proc. Engin. 150, 1056-1062 (2016)

30. I.B. Badriev, V.V. Banderov, V.L. Gnedenkova, N.V. Kalacheva, A.I. Korablev, R.R. Tagirov, Appl. Math. Sciences 9, 5697-5705 (2015) 